\title{
UCRL-TR-223809
}

LA W REN CE LIVERMORE N A TION A L LABORATORY

\section{Time-resolved Temperature Measurements in SSPX}

A. R. Ludington, D. N. Hill, H. S. McLean, J. Moller, R. D. Wood

August 18, 2006 
This document was prepared as an account of work sponsored by an agency of the United States Government. Neither the United States Government nor the University of California nor any of their employees, makes any warranty, express or implied, or assumes any legal liability or responsibility for the accuracy, completeness, or usefulness of any information, apparatus, product, or process disclosed, or represents that its use would not infringe privately owned rights. Reference herein to any specific commercial product, process, or service by trade name, trademark, manufacturer, or otherwise, does not necessarily constitute or imply its endorsement, recommendation, or favoring by the United States Government or the University of California. The views and opinions of authors expressed herein do not necessarily state or reflect those of the United States Government or the University of California, and shall not be used for advertising or product endorsement purposes.

This work was performed under the auspices of the U.S. Department of Energy by University of California, Lawrence Livermore National Laboratory under Contract W-7405-Eng-48. 


\section{Time-resolved Temperature Measurements in SSPX}

Final report on summer diagnostic project

by A.R. Ludington, U.S. Naval Academy under the Military Academy Research Associates Program and D.N. Hill, H.S. McLean, J. Moller, and R.D. Wood, Lawrence Livermore National Laboratory

August 4, 2006

This work was performed under the auspices of the U.S. DOE by the University of California, LLNL under contract No. W-7405-Eng-48. 


\section{Introduction:}

We seek to measure time-resolved electron temperatures in the SSPX plasma using soft X-rays from free-free Bremsstrahlung radiation. To increase sensitivity to changes in temperature over the range $100-300 \mathrm{eV}$, we use two photodiode detectors sensitive to different soft X-ray energies. The detectors, one with a $\mathrm{Zr} / \mathrm{C}$ coating and the other with a Ti/Pd coating, view the plasma along a common line of sight tangential to the magnetic axis of the spheromak, where the electron temperature is a maximum. The comparison of the signals, over a similar volume of plasma, should be a stronger function of temperature than a single detector in the range of $\mathrm{Te}<300 \mathrm{eV}$. The success of using photodiodes to detect changing temperatures along a chord will make the case for designing an array of the detectors, which could provide a time changing temperature profile over a larger portion of the plasma.

\section{Background:}

The soft X-ray diagnostic already in place on SSPX did not give any conclusive data about the changing temperature of the plasma along the chord the detector viewed. The correlation between the signal strength and the electron temperature measured through Thompson scattering was weak, if present at all. We looked at the expected signal as a function of $\mathrm{T}_{0}$, the electron temperature at the center of the plasma. The signal is the result of an integral over the volume of plasma whose radiation can reach the detector.

Total Signal $=\int_{V o l}\left[\Omega \cdot Q E \bullet E_{f f}(r)\right] d V$

where $\Omega$ is the solid angle of the detector, QE is the Quantum Efficiency of the detector as a function of photon frequency and $\mathrm{E}_{\mathrm{ff}}$ is the intensity of free-free Bremsstrahlung radiation per unit volume incident on the detector. $\mathrm{E}_{\mathrm{ff}}$ is solved for using another integral, over the range of incident photons. The intensity per unit volume per unit frequency interval is given by

$$
\frac{d E_{f f}}{d v}=C n_{e} n_{i} \overline{g_{f f}} \sqrt{\frac{\chi_{H}}{T_{e}}} e^{-\frac{h v}{T_{e}}} 1
$$

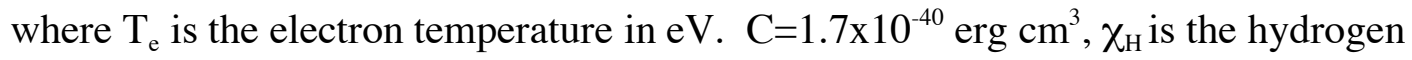
ionization potential of $13.6 \mathrm{eV}, \mathrm{g}_{\mathrm{ff}}$ is the Gaunt free factor (about 1), and $\mathrm{n}_{\mathrm{e}}$ and $\mathrm{n}_{\mathrm{i}}$ are electron and ion densities. Because we primarily want to compare detectors with a ratio of their signals and electron density is considered to be close to ion density, the equation can be written in a simpler form. We integrate over the values of photon frequencies and get

\footnotetext{
${ }^{1}$ T.F. Stratton "X-ray Spectroscopy," Plasma Diagnostic Techniques. Academic Press, New York, NY, 1996
} 
$Q E \cdot E_{f f}\left(T_{e}\right)=B \cdot \int_{\text {freq }} n^{2} Q E(v) \cdot T_{e}^{-\frac{1}{2}} e^{-\frac{h v}{T_{e}}} d v$

where B represents all the constants that fall out in the comparison. It also makes sense that electron temperature is not constant throughout the plasma. $T_{e}$ follows a relationship as a function of distance, $r$, from the magnetic axis.

$T_{e}(r)=\left(T_{0}-T_{\text {edge }}\right) \sqrt{1-\left(\frac{r}{a}\right)^{\alpha_{T}}}+T_{\text {edge }}$

where $T_{0}$ is the temperature at the magnetic axis of the plasma and $a$ is the toroidal radius of the plasma. The value $\alpha$ is chosen to be $1 . \mathrm{T}_{\text {edge }}$ is assumed to be zero. Density follows a similar relationship. Thus, we have

$T_{e}(r)=T_{0} \sqrt{1-\left(\frac{r}{a}\right)^{\alpha_{T}}} \quad$ and $\quad n(r)=n_{0} \sqrt{1-\left(\frac{r}{a}\right)^{\alpha_{n}}}$

which are included in the expression for $\mathrm{E}_{\mathrm{ff}}$ in the integral over volume.

Upon investigating the properties of the AXUV $100 \mathrm{Zr} / \mathrm{C}$ photodiode detector, the expected signal was seen to rapidly level off at a $T_{e}$ a little less than $200 \mathrm{eV}$. Because much of the Thompson data was greater than $200 \mathrm{eV}$, it is safe to say that the detector was unable to read any appreciable difference in soft X-ray level above $200 \mathrm{eV}$. The difference in signal would be about $10 \%$ between $200 \mathrm{eV}$ and $300 \mathrm{eV}$, which is too little to detect above the small fluctuations of the signal. To improve the performance of our measurement it was believed that we could implement a second detector. By choosing a detector that was sensitive to a different range of photon energies, we could compare the two signals. By taking the ratio of the two signals the constants drop out. If a change in the ratio of the two signals is observed, it should correspond to a change in $\mathrm{T}_{\mathrm{e}}$. Thus we had to find a detector which would be sensitive in the range of electron temperatures above $200 \mathrm{eV}$ and whose signal would change, with respect to that of the AXUV 100 $\mathrm{Zr} / \mathrm{C}$, by enough to be detectable.

\section{Method:}

The detector we found to suit our needs is the AXUV $100 \mathrm{Ti} / \mathrm{Pd}$. It's range of sensitivity complements that of sxr1. The configuration of SSPX is such that we would be able to install the two detectors on opposite sides of the same chord of plasma. This was hoped to make our comparison meaningful. Unfortunately the side on which the new detector was installed did not already have a viewing tube attached, so the viewing angles were not identical. On sxr2, as the new detector is called, we therefore installed a fixture which produces a different viewing angle, but the cross-section of plasma at the tangent point is close to that of sxr1. This was taken into account when the expected signal was calculated by integration over the volume in the detector's view. Exact measurements for each detector's geometry are included in Appendix A. 


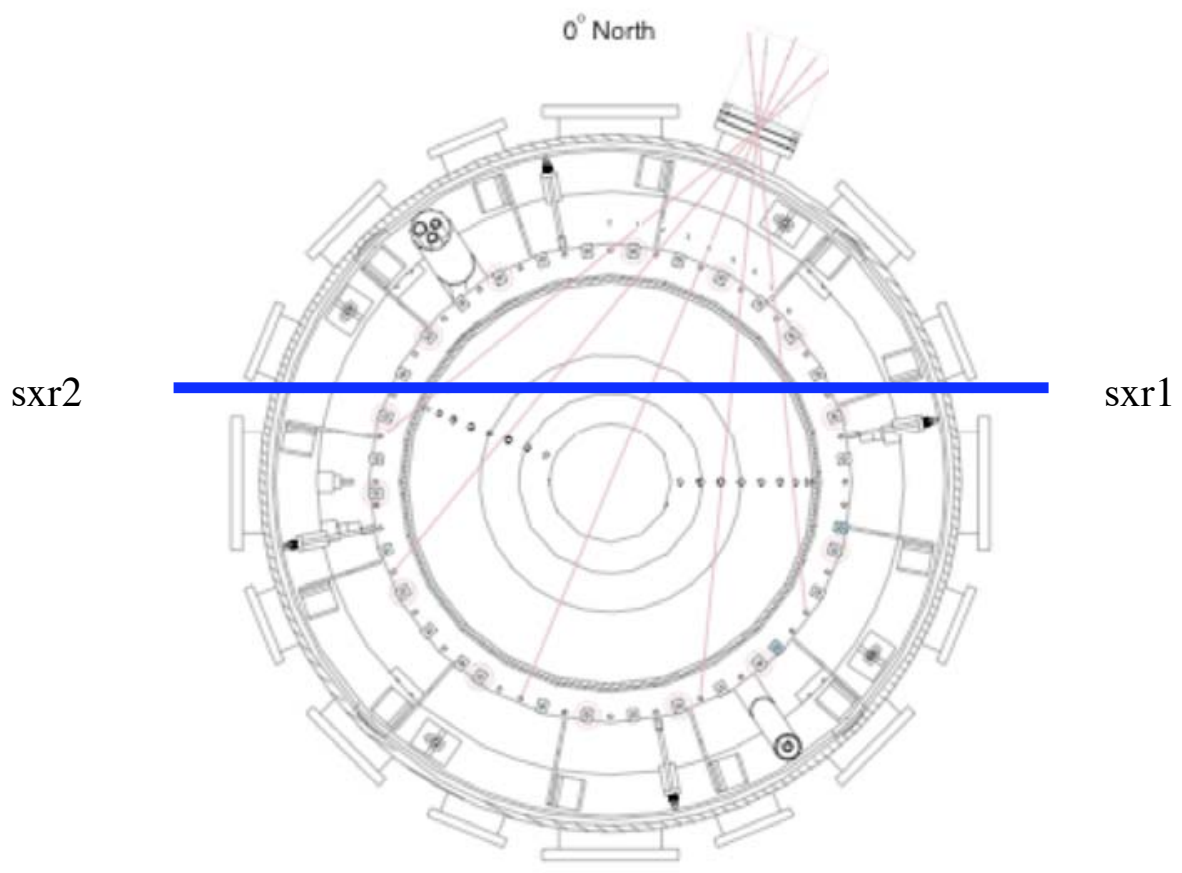

Figure 1. Overhead view of SSPX. Blue line indicates chord viewed by detectors

Using an excel spreadsheet and the table function, we integrated to find the magnitude of the signals we expected to see from each of these detectors. The ratio of these signals, at varying plasma temperatures is shown below. The comparison improves the diagnostic's ability to measure changes in temperature above $150 \mathrm{eV}$. There are no detectors available which will provide very good temperature resolution above $300 \mathrm{eV}$.

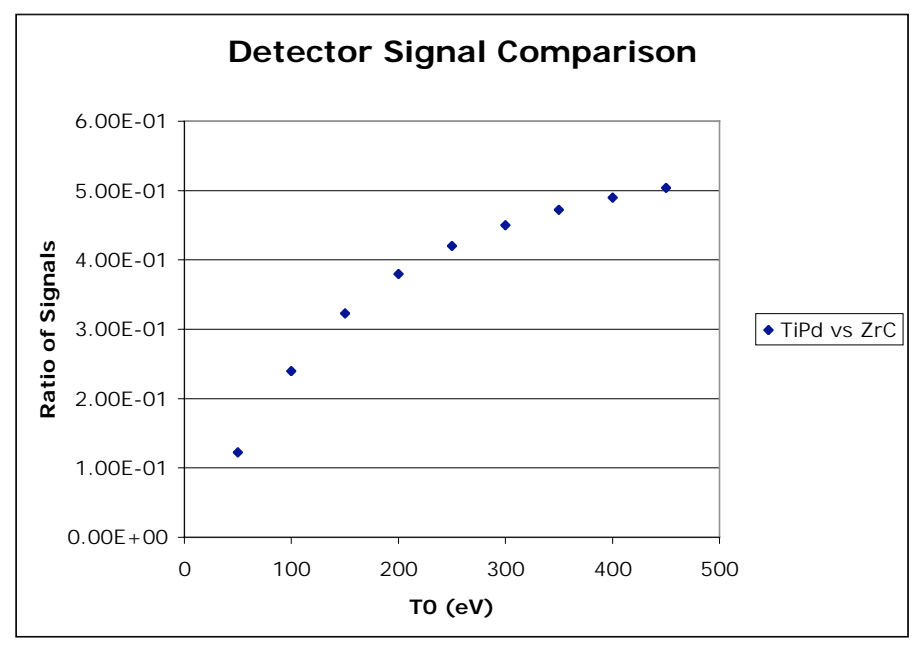


Figure 2. The expected ratio of the AXUV 1000 Ti/Pd detector (sxr2) to the AXUV 100 $\mathrm{Zr} / \mathrm{C}$ detector (sxr1) at varying plasma core temperatures

We varied the value of $\alpha_{n}$ and $\alpha_{T}$ to see what effect the changes in the temperature and density profiles had on the expected ratio. The effect was ignorable.

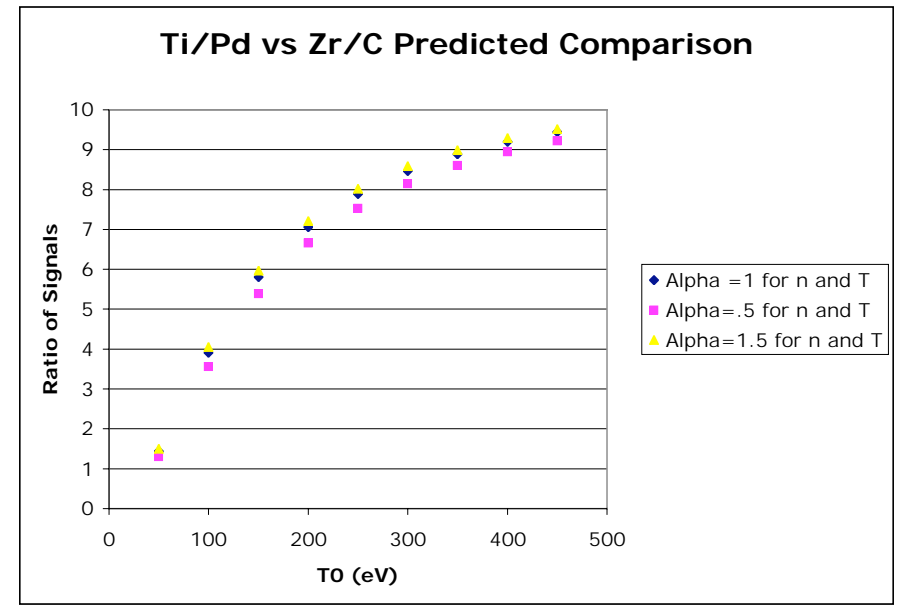

Figure 3. The signal comparison for differing temperature and density profiles. There is almost no difference.

We also were interested in what portion of the hot plasma we were viewing. So, for a $100 \mathrm{eV}$ plasma being viewed by sxr2, we performed the integral of the signal over varying distances from the magnetic axis. By plotting the results of these integrals against the value of their limits, we see that most of the signal comes from plasma within $8 \mathrm{~cm}$ of the magnetic axis.

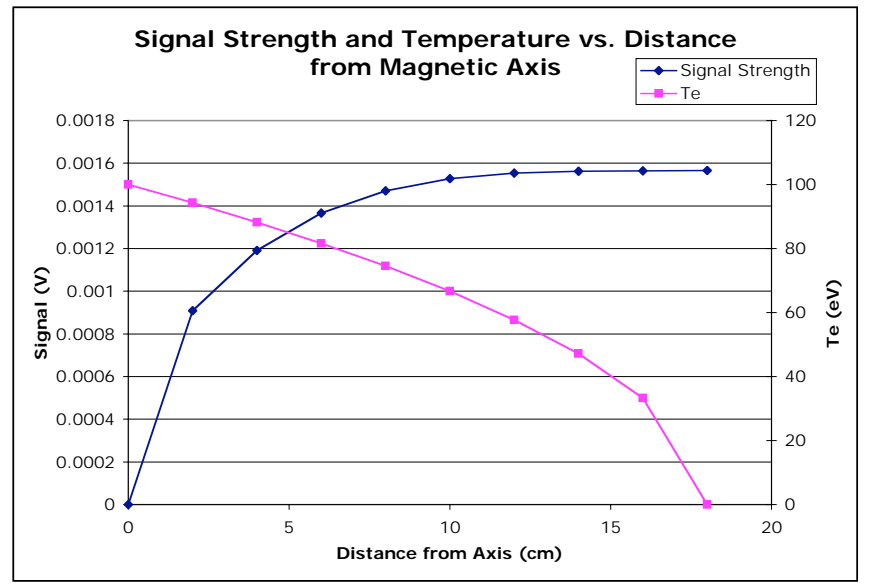

Figure 4. The signal when integrated over varying distances from the magnetic axis. Also plotted is the $\mathrm{T}_{\mathrm{e}}$ as a function of distance from the magnetic axis.

\section{Results:}

The initial results of sxr2 had a very low signal to noise ratio. This was easily remedied with an increase in the load resistor to $1 \mathrm{k} \Omega$. The ratio of the two signals showed a somewhat consistent behavior among the first few shots. However, the fluctuations in sxr1 were still large compared to the signal. For this reason we decided to 
increase the load resistor on $\operatorname{sxr} 1$ to $1 \mathrm{k} \Omega$ as well, in hopes that this would reduce noise and fluctuations. Below is data for some shots for which we used a $1 \mathrm{k} \Omega$ resistor on $\operatorname{sxr} 2$ and a $50 \Omega$ resistor on sxr1.

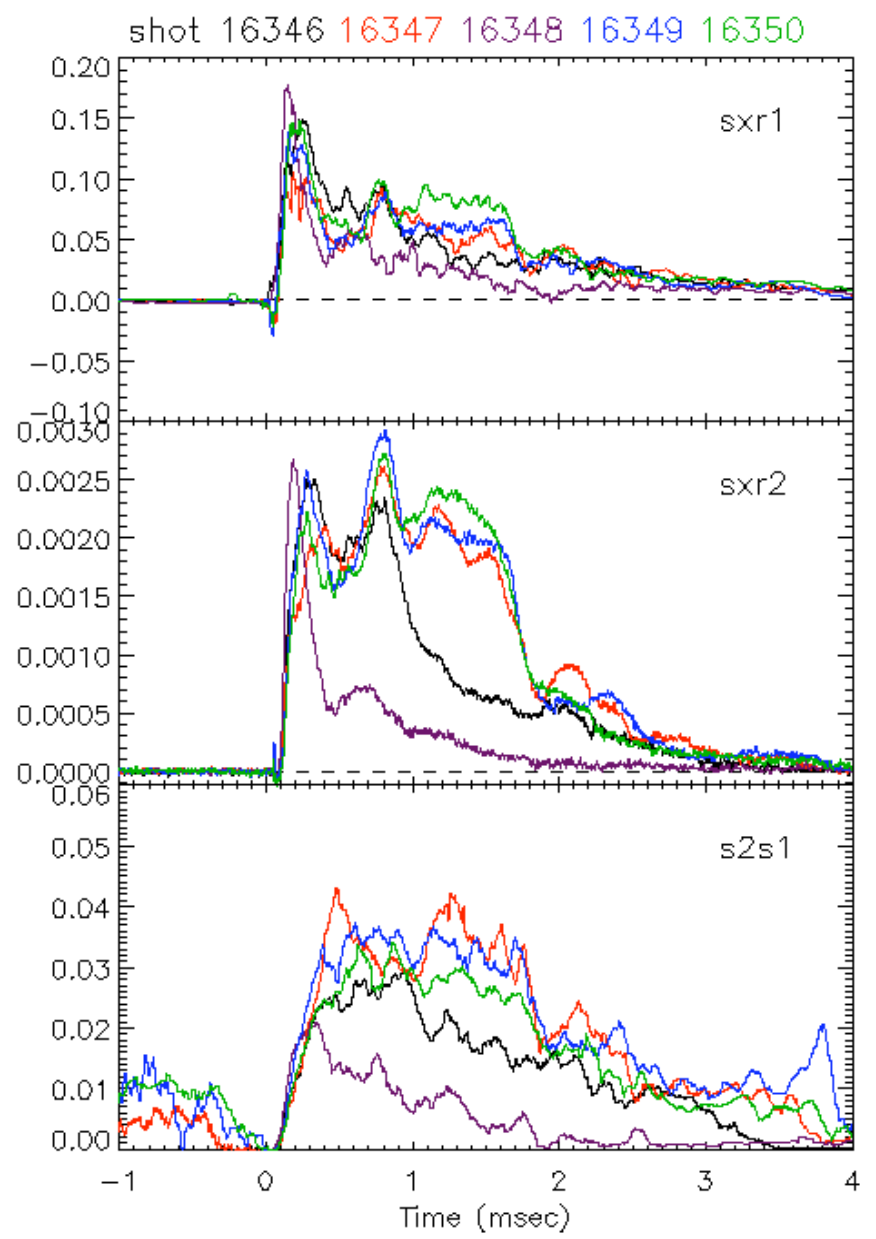

Figure 5. The signals from each detector, in Volts, along with the ratio between the two signals.

Putting a $1 \mathrm{k} \Omega$ resistor on sxr1 did not work because it caused us to saturate the channel, though the signal to noise ratio was increased. We were forced to go back to a $50 \Omega$ resistor on sxr1. A new variable, u_sxr_s2s1, was defined as the ratio between sxr2 and sxr1. This made it easy to call up our comparison. Results show large fluctuations in the signal ratio, and steep drops as well. The signal was, however, largely reproducible and the magnitude of the ratio corresponds to the data acquired by the Thompson data. The relationship between the ratio and electron temperature, at least when compared against Thompson, seems to be exponentially increasing though. We changed the configuration of the grounding on each channel so that, when finished both channels were grounded as sxr2 had been before. Appendix B diagrams these changes. 


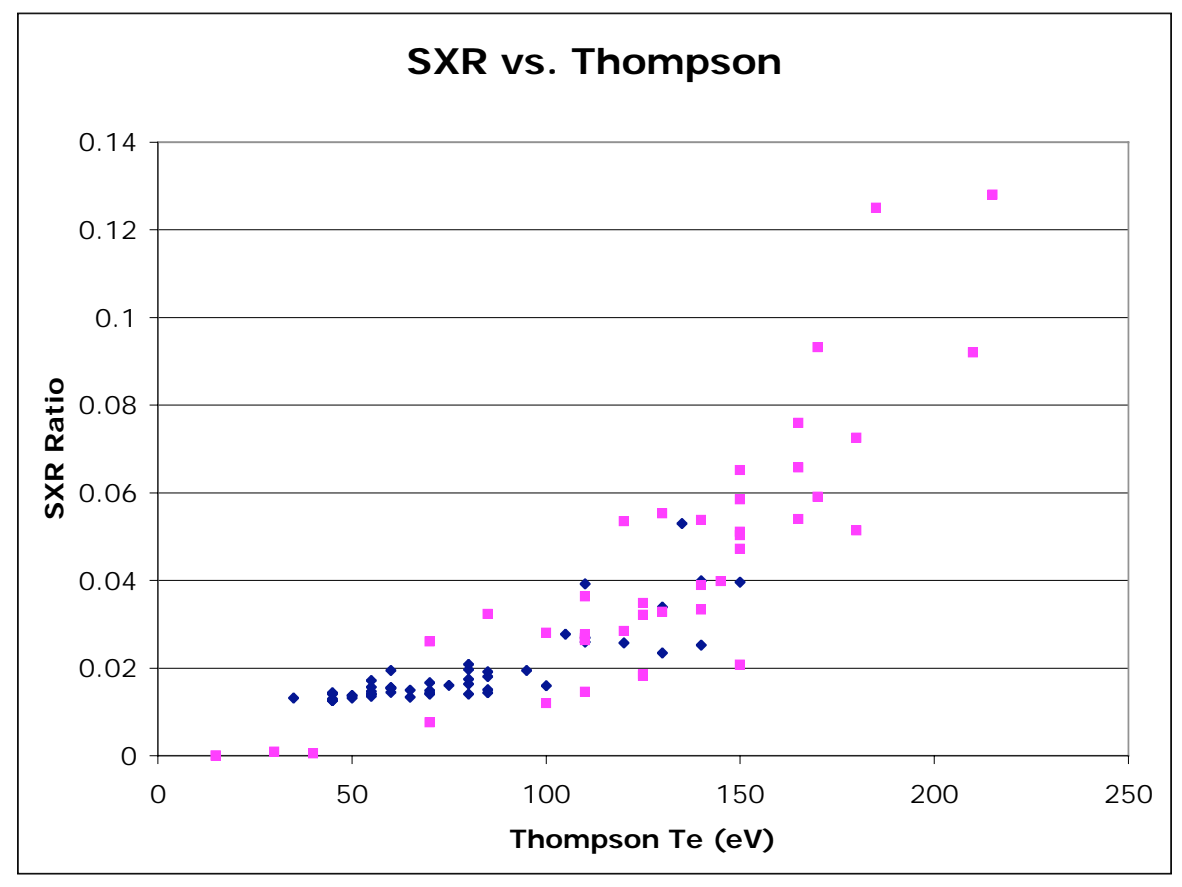

Figure 6. The soft X-ray signal ratio plotted against the Thompson measured temperature.

Some points (11 out of 116) with very large uncertainties in Thompson $\mathrm{T}_{\mathrm{e}}$ have been excluded. The blue data set has been normalized to fit the pink. The pink data was taken after the ground was added on sxr1.

\section{Discussion:}

Neutrals do not account for this behavior, because each detector would respond almost identically to the neutrals. Thus a significant neutral flux would flatten the curve of the signal ratio compared against temperature. Also, sxr1 is more sensitive to all the line radiation that we expected to see in the range of $10 \mathrm{eV}$ to $300 \mathrm{eV}$. Thus the ratio should be decreased by the presence of this radiation. It appears to be unchanged at the ionization potentials of any of the radiating ions. See Appendix $\mathrm{C}$ for the lines that are expected to be present. The fact that the soft X-ray ratio can be correlated to Thompson data is encouraging though. It was found to be reliable as a predictor. We noticed large jumps in SXR ratio at around $1.25 \mathrm{~ms}$ in shots 16393 to 16396, so the Thompson time was set earlier and it then measured higher temperatures, which fit our correlation. Thus we were able to predict higher temperatures at $1.25 \mathrm{~ms}$.

\section{Conclusion:}

The correlation between soft X-ray signal ratio and Thompson temperature gives us a good way to judge temperature fluctuations in the plasma through time. These results encourage the use of a detector array that could provide an even better comparison. The seemingly exponential rise in signal ratio cannot be explained by neutrals or line radiation. It may be the result of free-bound Bremsstrahlung or uncertainties in the integration of the expected signal. (We used an approximated field of view and a simplified profile of temperature and density.) Implementing an array of detectors could improve the comparison greatly. Also, other diagnostics like the CNPA, 
can provide information about the temperature of the plasma. For the time being, the soft $\mathrm{X}$-ray signal gives us a general idea of how the plasma temperature changes throughout a shot, and how those changes correlate to other qualities of the plasma. 


\section{Appendix A}

SXR 1: AXUV 100 Zr/C Photodiode, East Side



SXR 2: AXUV 100 Ti/Pd Photodiode, West Side

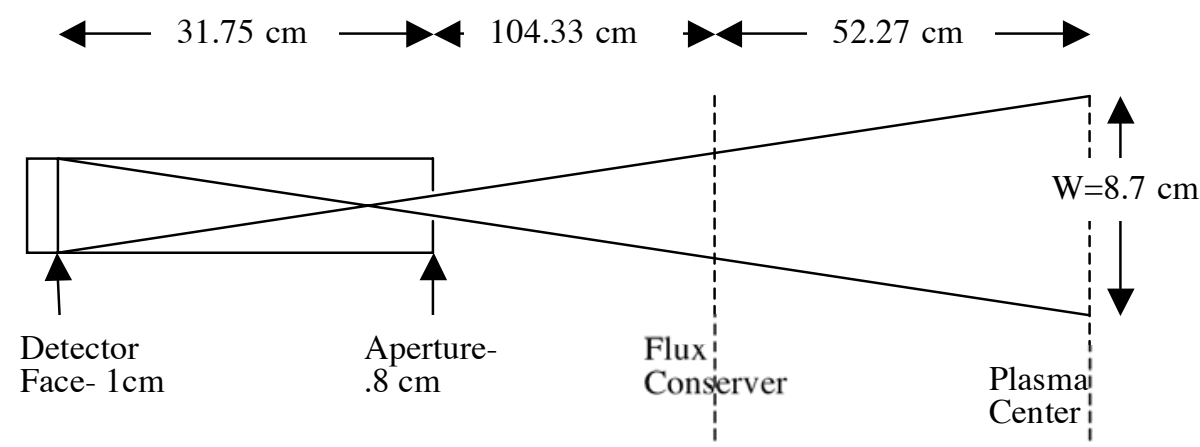

$\mathrm{W}$ indicates the width of the detectors field of view at the point tangent to the magnetic axis of the plasma. 


\section{Appendix B}

Changes to the grounding of sxr1 and sxr2

\begin{tabular}{|c|c|c|}
\hline Shot Number & SXR1 & SXR2 \\
\hline Prior to 16417 & OPEN & GROUND \\
\hline 16417 & OPEN & OPEN \\
\hline 16418 and all hence & GROUND & GROUND \\
\hline
\end{tabular}
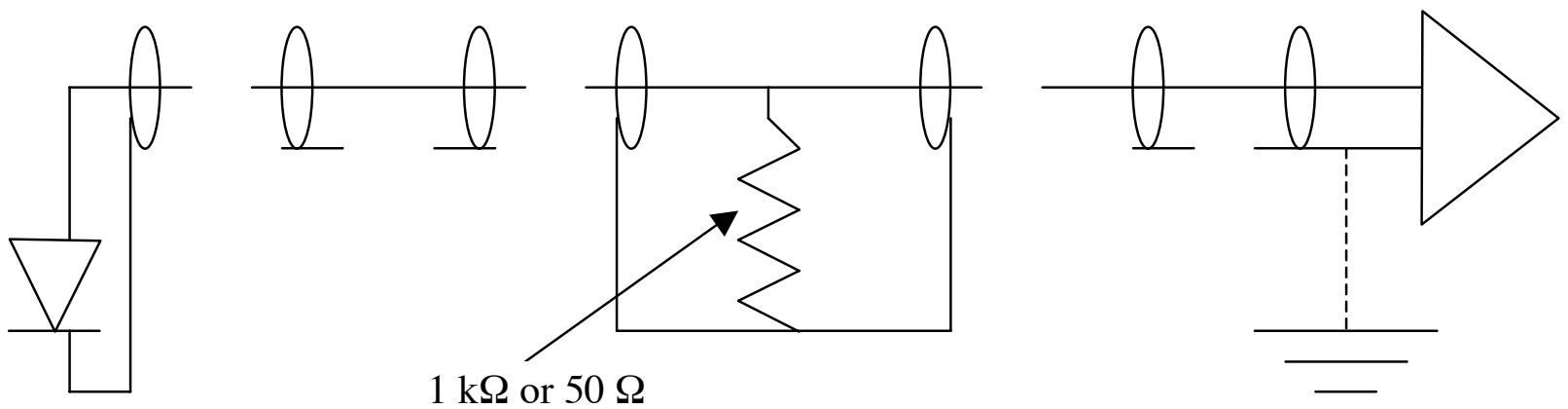

\section{Appendix C}

Line radiation expected for plasmas in SSPX

\begin{tabular}{|c|c|c|}
\hline Ion & Wavelengths $(\AA)$ & Ionization Potential $(\mathrm{eV})$ \\
\hline $\mathrm{C} \mathrm{V}$ & 40 & 392 \\
\hline O VI & 115 & 138 \\
\hline N VI & 29 & 552 \\
\hline Ti XI & $87.7,65$ & 265 \\
\hline Ti XII & $82.3,27$ & 299 \\
\hline Cu X & 87 & 232 \\
\hline Cu XII & 68,69 & 369 \\
\hline
\end{tabular}

Other radiation lines are from ions with very high ionization potentials, are not in the range of the detectors, are not very bright, or are from unlikely transitions. 